\title{
A Physically Plausible Model for Light Emission from Glowing Solid Objects
}

\author{
A. Wilkie ${ }^{1}$ and A. Weidlich ${ }^{2}$ \\ ${ }^{1}$ Charles University in Prague \\ ${ }^{2}$ RTT AG, Munich
}

\begin{abstract}
The emissive properties of glowing solid objects appear to be something that the graphics community has not considered in depth before. While the volumetric emission of plasma, i.e. flames, has been discussed numerous times, and while the emission characteristics of entire luminaires can be handled via IESNA profiles, the exact appearance of glowing solid objects appears to have eluded detailed scrutiny so far. In this paper, we discuss the theoretical background to thermally induced light emission of objects, describe how one can handle this behaviour with very little effort in a physically based rendering system, and provide examples for the visual importance of handling this in a plausible fashion.
\end{abstract}

Categories and Subject Descriptors (according to ACM CCS): I.3.7 [Computer Graphics]: Three-Dimensional Graphics and Realism-Color, shading, shadowing, and texture

\section{Introduction}

Over the past decades, the computer graphics community has invested considerable effort in deriving or adapting mathematical models for many physical effects that relate to the propagation of visible light in scenes, and its interaction with matter. This means that, for predictive rendering purposes, physically accurate, or at least very plausible, models are available for a wide variety of phenomena. However, somewhat surprisingly, the first stage of the lighting pipeline (emission $\rightarrow$ interaction with objects $\rightarrow$ perception), i.e. the actual processes that govern the emission of light from objects, seem to have received comparatively little attention within computer graphics so far.

Which is at least partially understandable, since for most graphics applications, modelling lightsources from first principles is not a sensible engineering approach. Even for Predictive Rendering tasks with demanding accuracy goals, using measured luminaire data [I1102] is usually a considerably more efficient (and saner) option than modelling the involved lamps down to individual lamp filaments (although this is in fact sometimes done to pre-calculate light distributions from lamps). But apart from luminaires, there is actually another class of light-emitting object that would, if one were after high degrees of visual accuracy, require an in-depth approach: glowing objects that emit energy in the visible range, such as pieces of iron that are being forged, flows of lava, or the exhaust of an engine running at power.

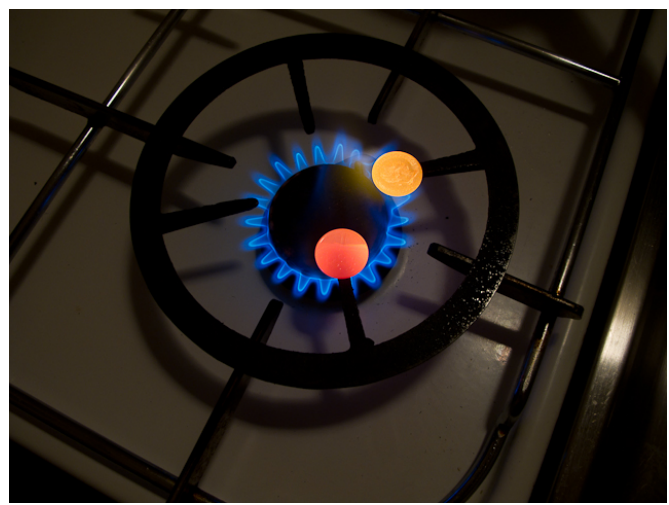

Figure 1: A photograph of two coins heated to approximately equal temperatures on a gas stove. At room temperature, the coin that glows red is silvery in appearance (nickelplated steel), while the coin that glows yellow-orange is made of gold. Note the considerable hue difference.

As can be seen in the photo in Figure 1, which shows two glowing coins heated by a gas flame, glowing objects at similar temperatures can exhibit quite dissimilar glow colours. 
What is not clearly visible in that photo is that the directional behaviour of the emission is also dependent on the surface finish of the involved object - a polished object will emit light differently from a diffuse one. Also, glowing objects can, under certain circumstances, emit partially polarised light - a photograph of this phenomenon can be seen in Figure 8. None of these aspects can be simulated with traditional measured data files for luminaires, or using a naive combination of unmodified blackbody radiation and a diffuse emission characteristic. But all three (hue, directional behaviour, polarisation) are potentially relevant for accurate simulations, such as those needed for luminaire design.

In this paper, we first discuss the physical background of glowing objects, discuss how such objects should be really modelled, and show the visual difference in colour, emission strength and polarisation this can make.

\section{Background and Related Work}

Current physically based rendering literature assumes that matter, when heated to a certain temperature, emits electromagnetic radiation of an exactly determined distribution and intensity [Gla95, PH04] - which is only true if the object in question is a blackbody in the physical sense. Also, in these publications, the emissive characteristics of a glowing object are apparently assumed to be diffuse. However, for real glowing objects, neither assumption is actually correct. In fact, the appearance of glowing objects is determined by two phenomena: the fundamental emission characteristics of matter at a given temperature (i.e. blackbody radiation), and the influence of the surface properties of the object's material on this basic emission spectrum (via Kirchhoff's law of thermal radiation).

The latter fact has actually been implicitly known in Computer Graphics for quite some time: both [HK93] and [DJ05] discussed scattering processes in layered materials. They both mention that the upwelling radiance which emerges from a scattering medium has to be attenuated in a way that is related to the reflectance properties of the material in question. Since the emission that emanates from a glowing object is also upwelling radiance from within, similar considerations have to apply. So the findings presented in this paper deal with the same underlying mechanism, albeit in a different context - previous graphics literature did not explicitly deal with upwelling radiance in self-luminous objects.

\subsection{Blackbody Radiation}

In physics, a blackbody is an idealised object that absorbs all electromagnetic radiation that falls on it [SH01]. For such an object, its temperature-dependent emission behaviour is comparatively easy to specify. Per unit solid angle it is, in dependence of wavelength $\lambda$ and temperature $T$, given by:

$$
I(\lambda, T)=\frac{2 h c^{2}}{\lambda^{5}} \frac{1}{e^{\frac{h c}{\lambda k T}}-1}
$$

As shown in Figure 2, with increasing temperatures this formula yields successively higher peak wavelengths for the resulting spectral emission distribution. The temperature at which matter starts to glow visibly is the so-called Draper point, at $798 \mathrm{~K}$. A group rendering of ideal blackbodies of increasing temperature can be seen in Figure 3.

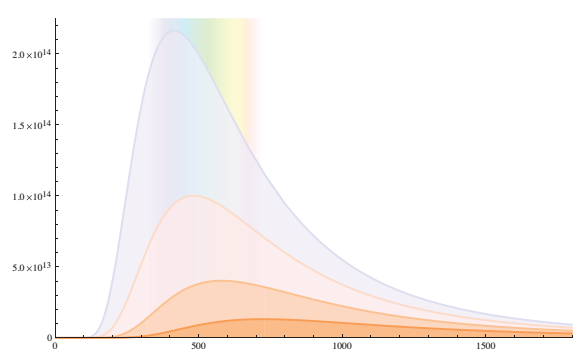

Figure 2: The spectral composition of blackbody radiation with increasing temperature, with the visible range indicated by the rainbow shaded area. The progression of the peak frequency towards shorter wavelengths with rising temperatures corresponds to the colour sequence seen in Figure 3.

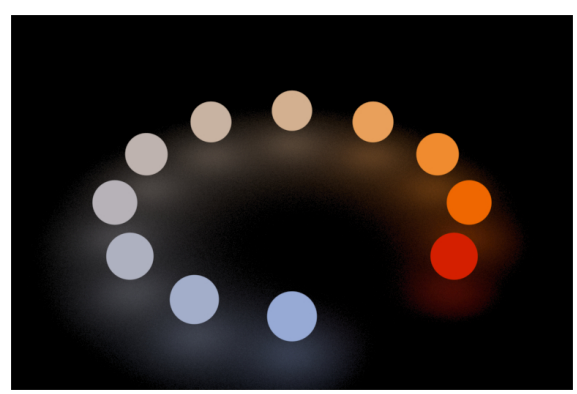

Figure 3: Rendering of a group of perfectly diffuse, neutrally coloured blackbody emitters. Temperature starts at $1000 \mathrm{~K}$, and rises in $500 \mathrm{~K}$ increments up to $5500 \mathrm{~K}$, with the last two at $6500 \mathrm{~K}$ and $8000 \mathrm{~K}$. The emission has been normalised, so they are of equal luminance; otherwise the difference in brightness between them would be huge.

\subsection{Kirchhoff's Law}

All objects that are at some non-zero temperature radiate electromagnetic energy. If they are a perfect black body, they diffusely radiate energy according to the blackbody radiation formula discussed in the previous section. However, black bodies are a theoretical concept, and rarely, if ever, encountered in nature. In reality, the emission of a solid, opaque object can be described as a simple extension of blackbody radiation, though. Kirchhoff's law of thermal radiation states that [SH01]:

At thermal equilibrium, the emissivity of an object equals its absorbance.

In this context, the absorbance is the fraction of incident light 
that is absorbed by the body/surface. A well-known corollary to this law is that

At a given temperature, no object can radiate more energy than an ideal blackbody at this temperature.

In other words, all real objects radiate less than an ideal blackbody - how much less only depends on their absorbance. This follows since blackbodies are, by definition, perfect absorbers - no object, real or theoretical, can absorb more. A practical consequence of this is that the lower the reflectivity of a real object, the closer it gets to blackbody behaviour, i.e. the higher its capability to lose energy via radiative emission. This is responsible for a lot of engineering decisions where the colour of an object is determined by the need to either retain or dissipate heat effectively, like e.g. the black colour of the underside of the Space Shuttle.

\subsection{Practical Relevance of Kirchhoff's Law in the Visible Range}

Kirchhoff's Law predicts that all coloured objects glow with hues that are different from the "standard" reddish-orangeyellow blackbody glow colour sequence associated with the phenomenon. As we can see in Figure 1, but also in the (idealised) simulation shown in Figure 7, there are cases where this behaviour can account for significant differences in appearance. But in general, colour changes compared to an "expected" glow colour, and markedly directional emission patterns, actually appear to be quite uncommon phenomena.

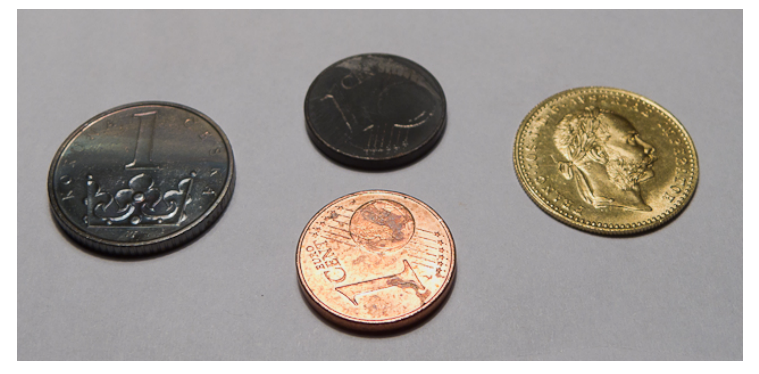

Figure 4: The upper three coins in this photo were heated in a gas flame, as shown in Figure 1. The left and middle coins (nickel-plated and copper-plated steel) exhibit marked oxidisation, which turned them both into neutrally coloured objects - and they both glowed with the same reddish hue. The gold coin on the right did not oxidise at all when held into the flame, and as a consequence, glowed with a markedly different hue from that of the others. The copper coin in front is there for comparison with the oxidised copper coin.

The main reason why the phenomenon is rarely noticed is that the majority of materials rapidly oxidise, as soon as they get very hot - and that this change is usually towards a colourless, diffuse appearance. If a shiny, reddish copper coin is held into a flame, it starts to glow, but it is at the same time also immediately covered by an opaque layer of black, diffuse cuprous oxide - which then diffusely emits radiation that is very close to unmodified Blackbody behaviour (see Figure 4 for a photo of coins that have been heated). And there is not even a need for flames to be present for oxidation to take place - for instance, molten iron oxidises very rapidly when in the presence of air. In some conditions, this can lead to a layer of rust particles floating on molten iron almost immediately after it is poured from a furnace. According to Kirchhoff's Law, such a layer of highly absorbing particles obviously strongly alters the emission characteristics of the glowing matter, and would have to be taken into account if a prediction of such a scene were desired. In this regard, noble metals such as gold are easier to handle, since no oxidisation takes place. This means that "abnormally" coloured glowing objects are not as common as Kirchhoff's law would suggest - but since there are plenty of scenarios, such as protective atmospheres, or thin, heat-resistant transparent coatings, which prevent oxidisation, any engineer working with Predictive Rendering should be aware of the phenomenon.

Also, there is at least one other phenomenon that affects the appearance of hot matter: the fundamental optical constants of substances, such as their complex index of refraction, change with temperature - usually also in the direction that the material becomes less colourful [Aks77].

\section{Modelling Glowing Surfaces in Computer Graphics}

In computer graphics, the options to describe the light $L$ received at a viewpoint $x$ from a point $x^{\prime}$ on a glowing object of temperature $T$ are (in order of increasing realism):

\subsection{Approach A - Diffuse Emission Only}

The simplest option, which we only mention here for completeness' sake, and because it seems to be implicitly assumed in some older literature [Gla95], is to assume a glowing object of temperature $T$ to be a diffuse emitter $I(T)$ :

$$
L_{A}(x, \omega)=I(T)
$$

Advantage: extremely simple, and easy to sample efficiently. Even though it is unrealistic (cf. fig. 3), this is still arguably the standard for area lightsources in a lot of GI rendering systems these days.

Disadvantages: noticeably unrealistic appearance. Since real glowing objects do not exhibit perfectly diffuse emission, no gradual transition from a cold (i.e. purely reflective) to a glowing state as seen in our test images is possible, and the colour of glowing objects can be quite wrong.

\subsection{Approach B - Simple Additive Combination of Reflectance and Emission}

If one wants to add the capability to describe the intermediate stages between cold and fully glowing objects, one only 
has to combine the integrated reflectance $\rho$ of the object in its cold state multiplied with the incident radiance $L^{\prime}$, and the blackbody radiation for the desired temperature $I(T)$ :

$$
L_{B}(x, \omega)=I(T)+\int_{\Omega} L^{\prime}\left(x^{\prime}, \omega^{\prime}\right) \cdot \rho\left(x, \omega, \omega^{\prime}\right) d \omega^{\prime}
$$

Advantages: simple, and gradual transitions between cold and glowing are possible. This is arguably one of the standard approaches when modelling weakly glowing, reflective objects in contemporary production CG.

Disadvantages: as with approach A, both the colour and the brightness of the glowing object can be wrong, as well as the directional behaviour of the radiation.

\subsection{Approach $\mathrm{C}$ - Combination According to Kirchhoff's Law}

The consequence of the physics background discussed in section 2 is that in order to describe a glowing object, one has to attenuate the blackbody radiation associated with its temperature with the absorbance $a$ of the surface at the point of interest. This attenuated energy is then used in exactly the same way as with approach B:

$$
L_{C}(x, \omega)=I(T) \cdot a(x, \omega)+\int_{\Omega} L^{\prime}\left(x^{\prime}, \omega^{\prime}\right) \cdot \rho\left(x, \omega, \omega^{\prime}\right) d \omega^{\prime}
$$

Since the conservation of energy dictates that the energy reflected by an object, and the energy absorbed by it, have to be equal to the flux of incident energy, the absorbance $a$ is easily computed as $1-f$ if one knows the reflectivity $f$ of an object.

Advantages: still simple (certainly not a lot more complicated than approach B), but considerably more realistic.

Disadvantages: none, at least in principle. There is one large caveat that goes with approach $\mathrm{C}$, though: obviously, the results obtained with such a technique are only as accurate as the BRDF model used for the reflectance $f$ of the glowing object. And in a lot of cases, this is a non-trivial problem as soon as very hot substances are involved (see section 2.3).

It is very important to note that the approach introduced in this section is not restricted to purely specular surfaces. The "one minus reflectance" constraint has to hold for all sorts of surfaces, so it directly applies to glossy or diffuse surfaces as well. In particular, microfacet-based BRDF models like Torrance-Sparrow can be immediately used together with this technique. The only reason we restricted the rendered images in this paper to perfectly smooth surfaces was in order to keep the number of test cases down.

\section{Results}

To demonstrate the effect that correct modelling of glowing surfaces can have, we concentrate on highlighting the difference between approaches $\mathrm{B}$ and $\mathrm{C}$ from section 3 - the very limited applicability of approach A is demonstrated by the unrealistic appearance of the spheres in Figure 3.

\subsection{Description of the Test Settings}

For our comparisons, we use opaque objects that are capable of being heated to temperatures where significant glow is noticeable without disintegrating, discolouring, or bursting into flame, i.e. we focus on at least theoretically realistic usage cases for predictive rendering purposes, such as the appearance of glowing or molten metal, and of glowing dielectrics such as glass or quartz with temperatures of up to $1600 \mathrm{~K}$. In this temperature range, the material constants which govern reflectance and absorbance (e.g. in the case of the Fresnel equations, the refractive index) are still reasonably close to their room-temperature values as they are usually quoted in literature. At higher temperatures, these do actually change, usually in the direction that the colourfulness of the material decreases [Aks77]. We also concentrate on opaque objects only because we want to discount volumetric effects, and discuss the surface appearance of glowing objects. But the principles of emission modelling discussed here also apply unchanged to transparent glowing substances, such as glass.

The scene we use contains only simple geometry spheres of increasing temperature, arranged in a circle on a diffuse base surface. Regardless of the material of the spheres, which changes between images, their arrangement is always the same: the coolest sphere, at the 11 o'clock position in the circle, is heated to $1050 \mathrm{~K}$, with $50 \mathrm{~K}$ increments in anti-clockwise direction - the hottest sphere at the 12 o'clock position is at $1600 \mathrm{~K}$. All spheres are assumed to be optically smooth, so their reflectance is governed by Fresnel reflectance [BW64].

We use the following materials as test cases: Silver as representative of a neutrally coloured, reflective metal, and copper and gold are examples of bright, coloured metals that affect the emission colour. Black quartz is a dielectric that is still solid at the given temperatures, and also of a material that is very dark (i.e. close to blackbody behaviour). Finally, selenium and platinum are used as intermediate cases of sorts - darker materials that consequently exhibit markedly stronger glow than their more shiny counterparts. Material constants were taken from literature [Pal85], and all images were rendered with a path tracer with 8 spectral samples.

\section{Simplifying Assumptions in the Test Settings}

The setting in which we place the glowing objects is idealised in two important respects: first, we assume a protective atmosphere that prevents oxidisation (see section 2.3) so that the surfaces of the objects retain their material composition regardless of temperature (incandescent light bulbs with their glowing metal filaments are filled with inert gas for this very reason). And secondly, the scenes shown in Figures 5, 6,7 and 10 are just a snapshot of a scene in which the various heated objects have been placed at the very moment the 


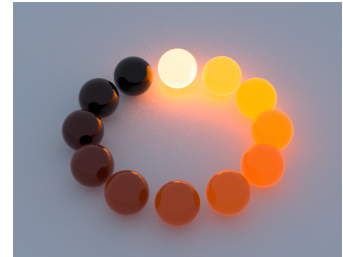

(a) Quartz, approach B

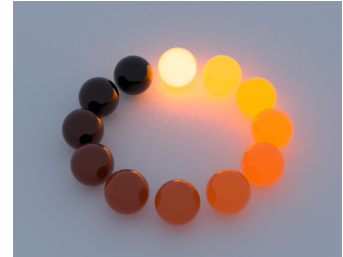

(c) Quartz, approach C

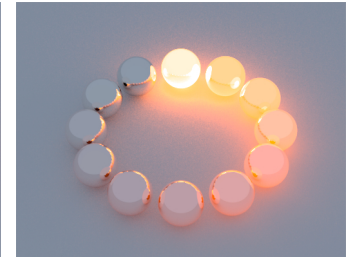

(b) Silver, approach B

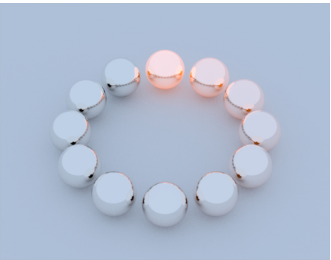

(d) Silver, approach C
Figure 5: The difference in glow intensity between modelling approaches B and C. The whitish glow of the hottest sphere in images a), b) and c) is a tone reproduction artefact due to its comparatively high brightness.

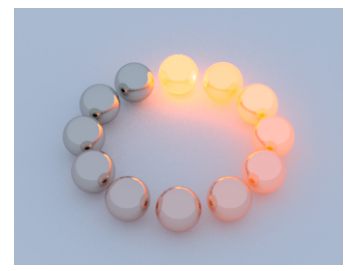

(a) Platinum

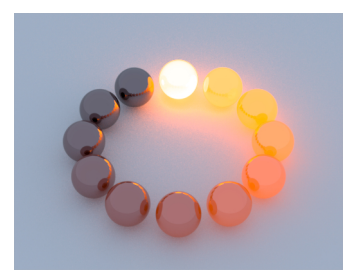

(c) Selenium

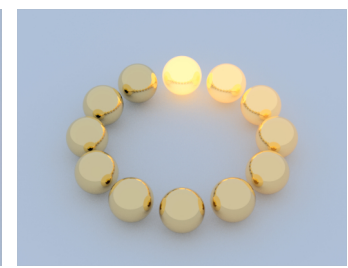

(b) Gold

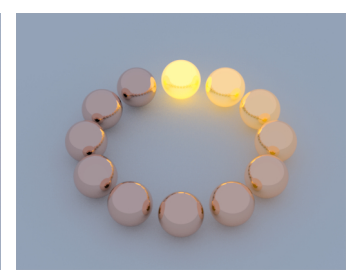

(d) Copper
Figure 6: The difference in glow intensity between various materials. Note the correspondence between the albedo of a material, and the intensity of the glow. The white sphere in c) is a tone reproduction artefact.

image is rendered. Without any external source of heat, the spheres would of course all rapidly cool down as time progressed, and decrease their emission. Moreover, they would of course also transfer heat between each other, and to the ground they are placed on. But neither of these two simplifying assumptions is relevant to demonstrate the nature and potential magnitude of the impact that the inclusion of Kirchhoff's law has on the modelling of glowing objects.

\subsection{Changes in Emission Strength}

The most noticeable effect of using approach $\mathrm{C}$ to model emission is that the brightness of the resulting glow can be significantly less than with approach B - this is in full accordance with the theory outlined in section 2 . Figure 5 shows a comparison between approaches $\mathrm{B}$ and $\mathrm{C}$ for two materials of very different albedo: black quartz and silver.

As expected, in the case of black quartz, no large differences between approach $\mathrm{B}$ and $\mathrm{C}$ are visible. The objects are relatively dark to begin with, and are therefore reasonable approximations of blackbodies. But note the discrepancies around the circumference of the spheres - if diffuse emission is simply added to the reflectance, this results in errors at the edges of the spheres. By contrast, the difference in brightness is very large for silver, in fulfilment of the "good reflectors are bad emitters" rule. However, good emitters do not automatically have to be dielectrics: selenium, a semiconductor which has a dark appearance in the visible range, glows almost as nicely as the black quartz specimen (see Figure 6).

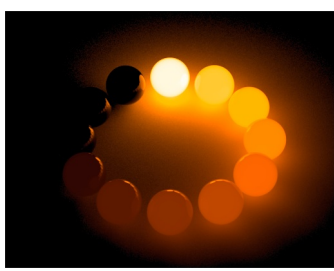

(a) Quartz

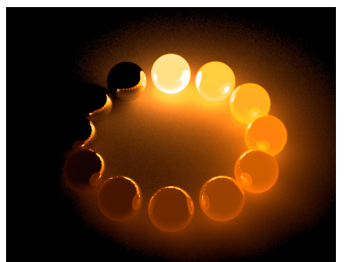

(c) Silver

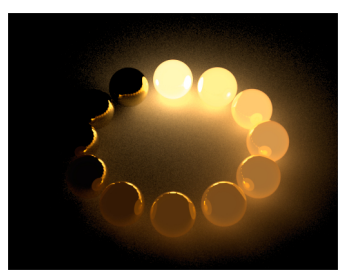

(b) Gold

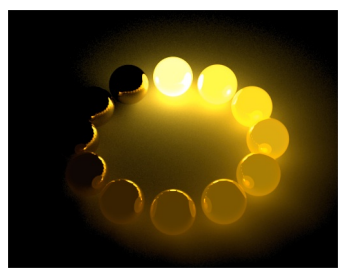

(d) Copper
Figure 7: Demonstration of how the absorbance of a glowing material affects glow colour. Note that without ambient illumination, the tone reproduction operator automatically reproduces the images at roughly similar brightness levels. Also, compare the colour of the gold and copper emissions with Figure 6 - there, the ambient illumination partially (but only partially) masks the altered emission hues.

\subsection{Changes in Emission Colour}

Figure 7 shows four images which demonstrate the colour changes that attenuation of the underlying blackbody radi- 


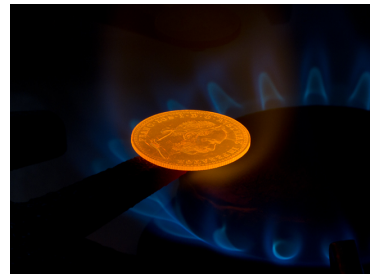

(a) $\leftrightarrow$ horizontal filter

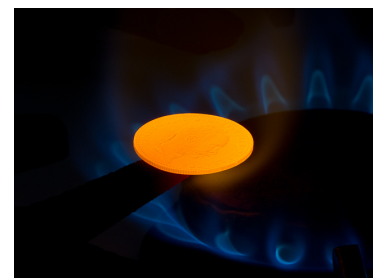

(b) $\uparrow$ vertical filter
Figure 8: Photograph of a polished, glowing gold coin taken through a linear polarisation filter. (a) a horizontally oriented filter cancels out part of the emission, while (b) a vertically oriented one enhances it. This is exactly the opposite effect that a linear polarisation filter has on reflected light, and consistent with the rendered results shown in section 4.4, and in particular those visualised in Figure 11.

ation with the absorbance of object material can cause. If approach B were used, all the spheres in these images would be of the same basic colour.

As expected, for the left two images (quartz and silver) the directional emission behaviour of the spheres is somewhat different, but the colour is roughly the same. The reflections from both silver and quartz are more or less neutrally coloured, so for rising temperatures the emission from both corresponds to a largely unmodified progression of blackbody colours. On the other hand, the emission from the glowing gold and copper spheres shows a markedly different hue than the reddish glow one would expect there. For coloured metals, this effect actually becomes even more pronounced at higher temperatures, where the underlying blackbody radiation contains more and more short wavelengths.

\subsection{Emission Polarisation}

A characteristic feature of thermally induced emission from smooth to moderately rough surfaces, which is totally lost if approach B is used, is that of emission polarization [San65, RPL99], a photograph of which can be seen in Figure 8. In the infrared region of the spectrum, where practically all objects emit some sort of "glow" even at room temperatures, the characteristic differences in polarisation behaviour between dielectric and conductors can be used to identify the basic material class seen in an IR image [WLT98], and the development of accurate models for the thermal emission polarisation of rough surfaces [AZ08] is an active research topic. These citations are just starting points into a very large and active field of research in the areas of thermal imaging and computer vision. Since both areas deal with electromagnetic radiation (albeit in different wavelength brackets), computer graphics can utilise some of the techniques developed there. Note that the issues described in section 2.3 also apply: the rapid oxidisation of many visibly glowing mate-
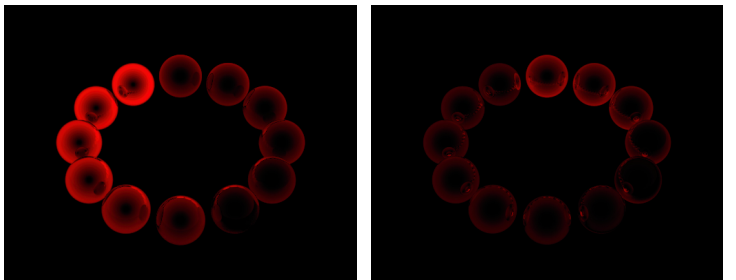

(a) Fig. 5c - Quartz, ambient(b) Fig. 5d-Silver, ambient light light
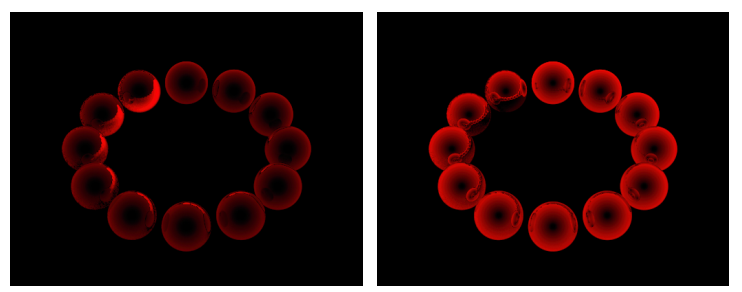

(c) Fig. 7a-Quartz, w/o ambient (d) Fig. 7c - Silver, w/o ambient
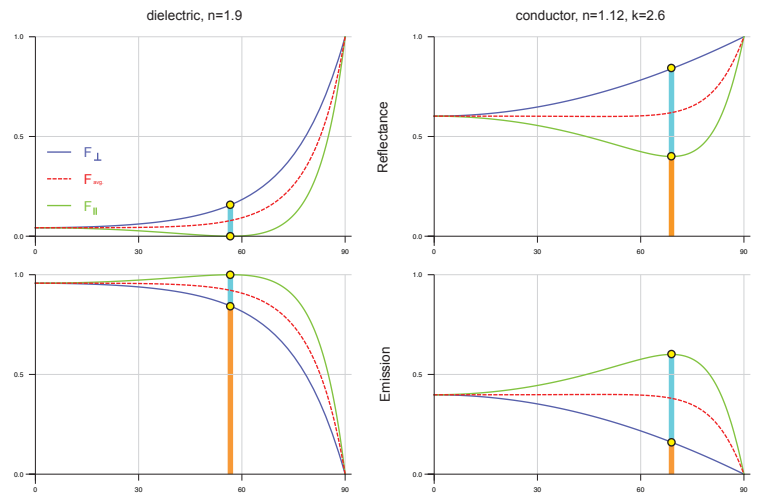

Figure 9: Top: Degree of polarisation plots [WW10] for images $5 \mathrm{c}, 5 \mathrm{~d}, 7 \mathrm{a}$ and $7 \mathrm{c}$. Left column: black quartz. Right column: silver. Note how reflected ambient light is capable of canceling out emission polarisation. Bottom: the corresponding reflection vs. emission plots. The lower set of curves is the inversion of the reflection functions: $1-F$. How much a given material polarises incident or exitant radiation is not solely determined by the difference between the two Fresnel components (cyan bars), but by the ratio of this difference to the unpolarised component of the interaction (orange bars). As a consequence, a dielectric that totally polarises reflected light at Brewster's angle will only emit weakly polarised light in that direction (plots on the left).

rials, along with the attendant increase in roughness, means that emission from visibly glowing objects is considerably less likely to be polarised than IR emission at room temperature. But non-reactive noble metals, such as gold, can be expected to exhibit considerable emission polarisation.

Since we used a polarisation-capable spectral rendering 


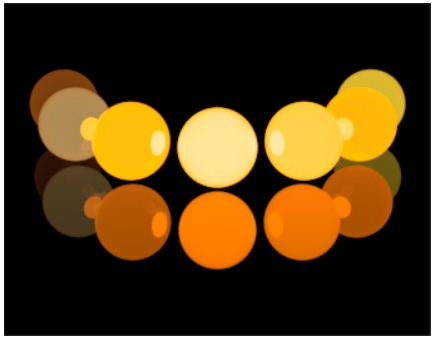

(a) No polarisation

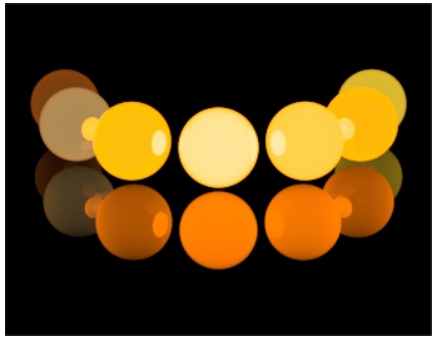

(b) With polarisation

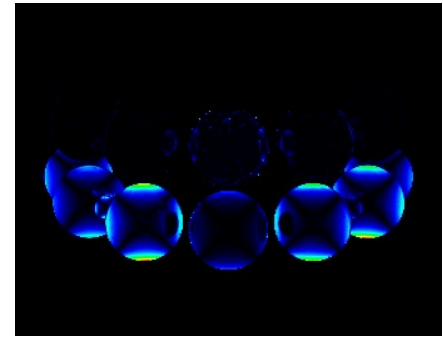

(c) CIE $L^{*} a^{*} b^{*} \Delta \mathrm{E}$

Figure 10: Reflections of glowing spheres in a black glass surface without (a) and with (b) polarisation taken into account. If polarisation is taken into account, the appearance of the reflections is noticeably altered. The weak differences on the glowing spheres themselves are due to direct inter-reflections, which are also slightly altered. (c) shows the difference image.

system to generate the test images in this paper, visualising the emission polarisation present in the test scenes is no extra effort. For the two Fresnel reflectance components $F_{\perp}$ and $F_{\|}$, the absorbance is computed separately as $1-F_{\perp}$ and $1-F_{\|}$; this automatically yields polarised emission.

Figure 9 shows the overall degree of polarisation for two materials - black quartz and silver - with and without ambient illumination. It is worth noting that the presence of ambient illumination of roughly similar strength to the glow of the objects (as seen in Figures 5 and 6) cancels out much of the intrinsic emission polarisation, and that the emission is only clearly polarised if the ambient illumination is switched off (as in Figure 7). The orientation visualisation of the linear emission polarisation shows that, as expected, it is rotated by 90 degrees relative to the reflection polarisation. Also note that one can clearly see something that is mentioned in polarimetry literature: the emission polarisation of metals is stronger than that of dielectrics because the reflective polarisation is weak, and vice versa. The plots in the lower half of Figure 9 demonstrate why this seemingly counter-intuitive behaviour is in fact directly determined by the behaviour of the Fresnel reflectance functions.

\subsubsection{Reflections of Glowing Objects}

One of the effects of this phenomenon can be seen in Figure 10. Since reflections from smooth phase boundaries are governed by the Fresnel terms which, amongst other things, also predict varying reflectance based on input polarisation, it stands to reason that the mirror image of a glowing object should look different when polarisation is taken into account. Figure 10 shows that under certain circumstances, this effect can be strong enough to be directly visible. This fact is important in the prototyping of e.g. lamps where a bright light source is put into a strongly reflective environment.

\subsubsection{Emission Characteristics of Incandescent Sources}

Apart from more or less weakly glowing objects like the ones discussed so far, it is also interesting to con- sider the polarisation characteristics of incandescent lightsources, i.e. very brightly glowing objects. The literature about this phenomenon goes in two directions: some authors attempt to characterise the emission polarisation of entire luminaires [Spo72, Kos80], while others attempt to measure the emission polarisation of a single glowing filament $\left[\mathrm{BCC}^{*} 09\right]$. The findings of the former two papers could be replicated in a polarisation-capable renderer, but most of the polarisation in that case would be due to interaction of the emitted light with the glass enclosure of the actual luminaire (i.e. polarisation via refraction and reflection).

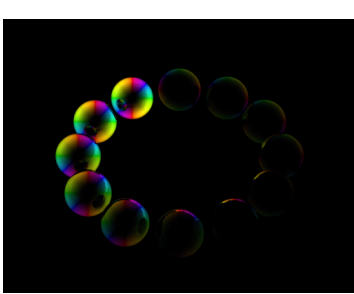

(a) Fig. 5c-Quartz, ambient

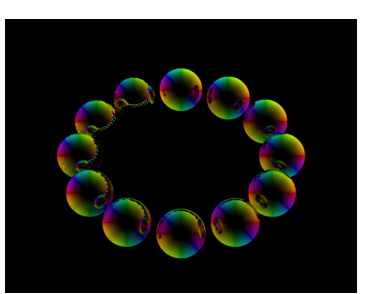

(b) Fig. 7c-Silver, dark
Figure 11: A plot of the direction of the overall linear polarisation (cf. [WW10]) found in some of the test images. Note that as expected, the orientation of the emission polarisation is rotated 90 degrees from that of the reflection polarisation.

More pertinent to our work is the latter paper, which deals with the emission of a single Tungsten filament. Their measurements, as well as their calculations, show that the diameter of the wire affects both the overall degree of emission polarisation, as well as its orientation. Apparently, the characteristics of strong incandescent emission from a thin wire can not be sufficiently described by application of Kirchhoff's law to a model of a long, thin, metallic cylinder. However, it is important to note that the orientation change of the emission polarisation for decreasing wire diameter (cf. Figure 1 in $\left[\mathrm{BCC}^{*} 09\right]$ ) takes place in a realm where other assumptions break down, and not necessarily the approach we 
present in sections 3.3 and 4.4. Recall that the Fresnel terms, which are normally used to model reflectance from smooth metallic objects in a graphics setting, are derived under the assumption that electromagnetic radiation is reflected from a planar phase boundary of infinite extent. This assumption is obviously not met for a cylinder with a diameter on the same order of magnitude as the wavelength of light. In this case, a different mathematical approach [BCC $\left.{ }^{*} 09\right]$ which is capable of yielding variable emission polarisation dependent on the wire diameter is needed instead.

For larger wire diameters, the behaviour reported in literature $\left[\mathrm{BCC}^{*} 09\right]$ actually agrees with our results; this scenario is referred to as the "thick wire case". If one uses the formulas presented in section 4.4 on an emissive cylinder, the linear polarisation direction is correct: the reflective polarisation caused by a cylinder is aligned with the cylinder axis, so the emission polarisation is orthogonal to it - which again is in agreement with measurements found in cited literature.

\subsubsection{Capturing Polarisation Profiles}

As noted earlier, it is usually not feasible to model lamps down to the level of individual filaments when it comes to simulating light transport in a large, generic scene. But it is worth noting that the emissive polarisation profile of a lightsource (such as the patterns seen in [Spo72]) could easily be included in a generalised extended luminaire description format. The contents of such profiles could either be measured, or precomputed using the method outlined in this paper.

Such an approach would allow the inclusion of the effects described in this paper without having to deal with the inner workings of each luminaire during rendering. For Predictive Rendering purposes, this attention to detail can be necessary in some cases: emission polarisation information can be essential when trying to accurately predict the appearance of scenes that include many specular interreflections [WUT* ${ }^{*}$ ]].

\section{Conclusion and Outlook}

We demonstrated the benefits of using Kirchhoff's law of thermal emission and its influence on emission colour, strengths and polarization to derive a physically plausible description of glowing surfaces. In terms of procedure, the difference to the state of the art (i.e. simple addition of the reflective and emissive components) might not seem large, but the proper combination proposed in this paper yields significant improvements in the capability to predict the appearance of glowing objects. Also, while all results in this paper were generated with an offline spectral renderer, nothing precludes use of this technique in realtime rendering settings.

As noted in sections 2.3 and 3, the "1 minus reflectance" approach to attenuating blackbody emission presented in this paper has one potential achilles heel that is rooted in the nature of the problem: an accurate description of reflectance for hot objects can, under certain circumstances, and for certain materials, require quite different models than those needed at room temperature. This potential complication does not, however, invalidate the fundamental approach presented in this paper. In the future, we plan on investigating this aspect further, and to derive models for complex molten substances.

\section{References}

[Aks77] AKsyutov L. N.: Temperature dependence of the optical constants of tungsten and gold. Journal of Applied Spectroscopy 26 (1977), 656-660. 10.1007/BF00611851.

[AZ08] AN C., ZERINGUE K.: Emission polarization from rough surfaces. Polarization: Measurement, Analysis, and Remote Sensing VIII 6972, 1 (2008), 697200.

[BCC*09] Bimonte G., CAPpellin L., CARugno G., Ruoso G., SAADEH D.: Polarized thermal emission by thin metal wires. New Journal of Physics, 11 (2009).

[BW64] Born M., Wolf E.: Principles of Optics. The Macmillan Company, 1964.

[DJ05] DONNER C., JENSEN H. W.: Light diffusion in multilayered translucent materials. ACM Transactions on Graphics 24, 3 (July 2005), 1032-1039.

[Gla95] GLASSNER A. S.: Principles of digital image synthesis. Morgan Kaufmann Publishers, San Francisco, California (1995).

[HK93] HanRAhan P., KRUEGer W.: Reflection from layered surfaces due to subsurface scattering. In SIGGRAPH (1993), ACM, pp. 165-174.

[I1102] ILluminating ENGINEERING SocIETy: ANSI/IES LM63-02: ANSI Approved Standard File Format for Electronic Transfer of Photometric Data and Related Information, 2002.

[Kos80] Kostuk R. K.: Polarization characteristics of dxw-type filament lamps. Applied Optics 19, 14 (1980).

[Pal85] PALiK E. D.: Handbook of Optical Constants of Solids. Academic Press, 1985.

[PH04] Pharr M., Humphreys G.: Physically Based Rendering: From Theory to Implementation. Morgan Kaufmann Publishers Inc., San Francisco, CA, USA, 2004.

[RPL99] RESNiCK A., PERSONS C., Lindquist G.: Polarized emissivity and Kirchhoff's Law. Appl. Opt. 38, 8 (1999), 13841387.

[San65] Sandus O.: A review of emission polarization. Appl. Opt. 4, 12 (1965), 1634-1642.

[SH01] Siegel R., Howell J. R.: Thermal Radiation Heat Transfer, 4th Edition. Taylor \& Francis, New York, NY, 2001.

[Spo72] SPOONER D.: Polarization pattern of a high intensity incandescent lamp. Applied Optics 11, 12 (1972).

[WLT98] WolfF L. B., Lundberg A. J., TANG R.: Image understanding from thermal emission polarization. In Image Understanding Workshop (1998), pp. 747-754.

[WUT*04] Wilkie A., Ulbricht C., Tobler R. F., Zotti G., PURGATHOFER W.: An analytical model for skylight polarisation. In Eurographics Symposium on Rendering (Norrköping, Sweden, 2004), Keller A., Jensen H. W., (Eds.), Eurographics Association, pp. 387-397.

[WW10] WILKIE A., WEIDLICH A.: A standardised polarisation visualisation for images. In Proceedings of the Spring Conference on Computer Graphics 2010 (SCCG 2010) (May 2010). 